\title{
An Oscillatory Short-Term Memory Buffer Model Can Account for Data on the Sternberg Task
}

\author{
Ole Jensen and John E. Lisman \\ Volen Center for Complex Systems, Brandeis University, Waltham, Massachusetts 02243
}

A limited number $(7 \pm 2)$ of items can be held in human short-term memory (STM). We have previously suggested that observed dual (theta and gamma) oscillations could underlie a multiplexing mechanism that enables a single network to actively store up to seven memories. Here we have asked whether models of this kind can account for the data on the Sternberg task, the most quantitative measurements of memory search available. We have found several variants of the oscillatory search model that account for the quantitative dependence of the reaction time distribution on the number of items (S) held in STM. The models differ on the issues of (1) whether theta frequency varies with $S$ and (2) whether the phase of ongoing oscillations is reset by the probe. Using these models the frequencies of dual oscillations can be derived from psycho- physical data. The derived values $\left(f_{\theta}=6-10 \mathrm{~Hz} ; f_{\gamma}=45-60 \mathrm{~Hz}\right)$ are in reasonable agreement with experimental values. The exhaustive nature of the serial search that has been inferred from psychophysical measurements can be plausibly explained by these oscillatory models. One argument against exhaustive serial search has been the existence of serial position effects. We find that these effects can be explained by short-term repetition priming in the context of serial scanning models. Our results strengthen the case for serial processing and point to experiments that discriminate between variants of the serial scanning process.

Key words: theta; gamma; oscillations; working memory; short-term memory; Sternberg; brain waves
The brain has a limited short-term memory (STM) capacity ( $7 \pm$ 2 items) for list items, such as the digits in a novel phone number (Brener, 1940; Miller, 1956). If human STM is maintained by active firing as observed in prefrontal cortex of the monkey (Goldman-Rakic, 1995; Fuster, 1997), there is a fundamental problem of how to keep multiple memories separate. It has been suggested that this is accomplished by a "buffer" (Atkinson and Shiffrin, 1968), but the physiological mechanisms that would allow multiple items to be stored in a buffer are not known. We have proposed (Lisman and Idiart, 1995; Jensen et al., 1996) that a single brain network can separately maintain up to seven memories by a multiplexing mechanism that uses theta (Gundel and Wilson, 1992; Mecklinger et al., 1992; Nakamura et al., 1992; Iramina et al., 1996; Krause et al., 1996; Sasaki et al., 1996; Gevins et al., 1997; Klimesch et al., 1997; Tesche, 1997) and gamma (Galambos et al., 1981; Pantev et al., 1991; Joliot et al., 1994; Tallon-Baudry et al., 1997, 1998) brain oscillations for clocking. A memory is represented by groups of neurons that fire in the same gamma cycle. Individual memories become serially active in sequential gamma subcycles of a theta cycle (Fig. 1). This pattern of activation repeats on subsequent theta cycles. We have previously shown that a multiplexing buffer of this kind can be plausibly realized by known biophysical mechanisms (Lisman and

Received June 9, 1998; revised Sept. 25, 1998; accepted October 1, 1998.

This work was supported by National Science Foundation Grant IBN-9723466 and the Alfred P. Sloan Foundation. We gratefully acknowledge the support from the W. M. Keck Foundation. We thank Michael Kahana (Brandeis University) and Saul Sternberg (University of Pennsylvania) for many helpful discussions. Saul Sternberg has kindly provided us with his data on the moments of the RT distributions (Sternberg, 1964), and Greg Ashby (University of California, Santa Barbara) and Jerry Balakrishnan (Purdue University) have provided us with the full data set of the RT distributions (Ashby et al., 1993).

Correspondence should be addressed to Dr. John E. Lisman, Volen Center for Complex Systems, Brandeis University, Waltham, MA 02243.

Copyright (C) 1998 Society for Neuroscience $\quad 0270-6474 / 98 / 1810688-12 \$ 05.00 / 0$
Idiart, 1995; Jensen and Lisman, 1996a; Jensen et al., 1996). A key underlying idea is that a memory is represented by cells firing within a gamma cycle, and different memory representations are activated in different gamma cycles. Recent work by Wehr and Laurent (1996) is consistent with the idea that gamma oscillations serve as a clock for information processing. They showed that components of sequences representing odors are active in successive gamma cycles. Furthermore, modeling work shows that the phase advance of hippocampal place cells can be quantitatively explained in terms of expected positions read out in sequential gamma subcycles of a theta cycle (Jensen and Lisman, 1996b).

To further test the concept of a multiplexing buffer, we have asked whether models of this kind can account quantitatively for data from the Sternberg task (Sternberg, 1966). This task has been extensively used to explore the timing of retrieval from STM, and there is general agreement about the central findings. In the task, a list of items is presented rapidly. A few seconds later, a probe item is presented, and the subject answers as quickly as possible whether the probe was on the list. A key finding consistent with serial memory scanning is that the average reaction time (RT) increases linearly with the number of items on the list (S). A second key finding is that the increase in RT with $\mathrm{S}$ is the same for "yes" (positive probes) and "no" (negative probes) answers. This observation led to the suggestion that the search is exhaustive (Sternberg, 1966): the answer can apparently not be given until the entire list is scanned.

In our initial effort to link STM to brain oscillations we pointed out that the increase in RT with each additional item (the "slope" of the Sternberg curves) approximates the period of one gamma cycle (Lisman and Idiart, 1995). Furthermore, the number of gamma subcycles that occur during a theta cycle (Bragin et al., $1995)$ is close to the human memory span of $7 \pm 2$. It was these correspondences that suggested that an oscillatory model based 


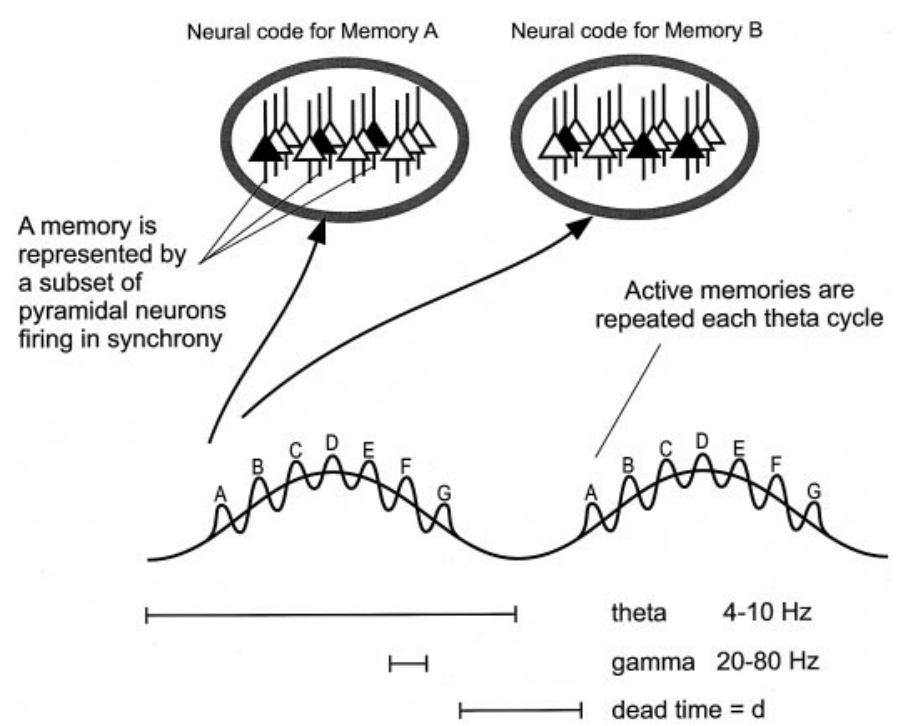

Figure 1. Seven memories $(A-G)$ are multiplexed; memory $A$, represented by a certain spatial pattern of cell firing (oval inset), is active in the first gamma subcycle of a theta oscillation, followed by memory $B$ in the next gamma cycle, etc. After a dead time $(d)$, the seven memories repeat in the subsequent theta cycle.

on theta and gamma oscillations might organize STM. However, we did not show that an oscillatory model could quantitatively explain the full RT distributions and their dependence on S. This is one of the goals of the current study.

A second goal has been to analyze one of the major objections to serial scanning. A simple exhaustive scanning model would predict that the RT is the same for all items stored in STM. However, a strong serial position effect has been found if the retention interval between the presentation of the list and the probe is $<1.5 \mathrm{sec}$ (Clifton and Birenbaum, 1970; Burrows and Okada, 1971; Forrin and Cunningham, 1973): subjects respond faster to items at the end of the list. We have found that this data can be simply explained by short-term repetition priming in the context of a serial scanning model.

\section{RESULTS}

We have sought to find oscillatory models that can account quantitatively for the details of RT distributions in the Sternberg task. As found by Sternberg (1966), the linear increase in RT with memory load ( $\mathrm{S}$ ) is $\sim 38 \mathrm{msec} /$ item (Fig. 2). The increment is the same for negative and positive probes. Sternberg (1964) also found that not only does the RT increase with S, but the variance and asymmetry (measured by the third central moment) also increase with S (Fig. 2). The cumulative RT distributions have been characterized by Ashby et al. (1993). As seen in Fig. 3, the cumulative distributions are systematically delayed as $\mathrm{S}$ gets larger. Thus the mean RT increases with S. A final important feature of the Sternberg data is the effect of serial position: subjects respond faster to a probe that corresponds to an item late in the list (Forrin and Cunningham, 1973). However, when the delay between the presentation of the list and the probe becomes sufficiently long $(>1.5 \mathrm{sec})$, the serial position effect vanishes (see Fig. 10).

The oscillatory models that we have considered are all based on the following principles: a memory is represented by a subset of neurons firing within a gamma cycle (Fig. 1). When multiple memory representations are being kept active during a retention
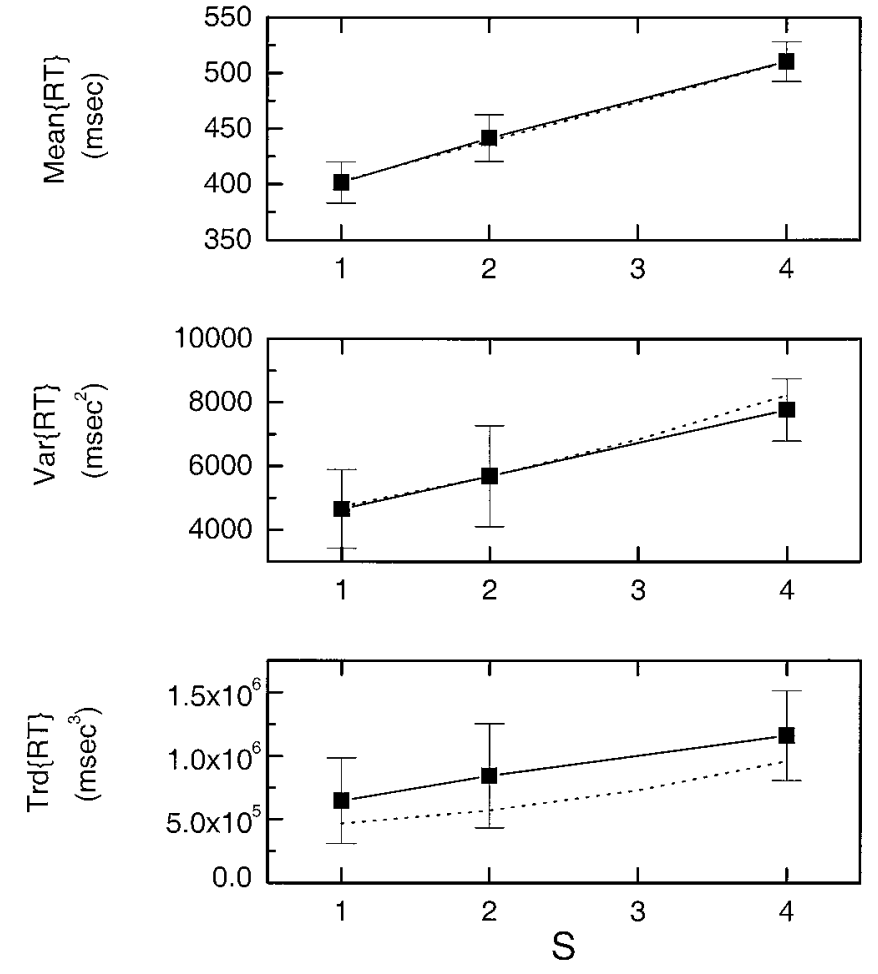

Figure 2. Adapting theta model fit (dashed lines) to experimental data (solid lines) for mean, variance, and skewness of the RT distribution for the Sternberg task. The data obtained by Sternberg are responses to negative probes only. The best fit using Equations 13, 15, and 16 resulted in the parameters $T_{\gamma}=22 \mathrm{msec} ; p_{\mathrm{a}}=0.88 ; d=80.1 \mathrm{msec} ; \tau_{\text {motor }}=57$ msec; and $t_{0}=215 \mathrm{msec}$. The fit was obtained by first fitting the slopes of the increases with $\mathrm{S}$ and then fitting $\tau_{\text {motor }}$ and $t_{0}$. Error bars indicate SEs. Data are average of 10 subjects.

period, they are activated in successive gamma subcycles of a theta cycle. This pattern repeats during each theta cycle (Fig. $4 A$ ). We have previously implemented a physiologically plausible network model of such a buffer (Lisman and Idiart, 1995; Jensen and Lisman, 1996a; Jensen et al., 1996). This model is constructed of a network of excitatory and inhibitory cells. The excitatory cells receive an external oscillatory drive at theta frequency. Each of the excitatory cells have the following properties: a cell will remain inactive until it is activated by an external input triggering an action potential. After the action potential follows a depolarizing ramp (afterdepolarizing potential), which repeatedly brings the cell to fire in subsequent theta cycles. This allows the storage of a memory representation by repeated activation at theta rate. Each time a memory representation is activated the inhibitory cells in the network provide a hyperpolarizing feedback. This feedback, which produces the gamma oscillations, serves to keep multiple memory representation separate in time. Recurrent collaterals have synaptic weights that encode each item (but not the unique sequence in the list) and enable the network to reactivate memories in the correct sequence, even in the presence of noise.

The RT in the Sternberg task can be described as the sum of various components (Fig. $4 A$ ). In the Sternberg paradigm a probe typically arrives $500-3000 \mathrm{msec}\left(t_{\text {retention }}\right)$ after the end of item presentation (typically $0.5-1.2 \mathrm{sec} /$ item). This probe then has to be identified. After the item is identified, we assume that there is a wait period $\left(t_{\text {wait }}\right)$ until the beginning (trough) of a theta cycle. 

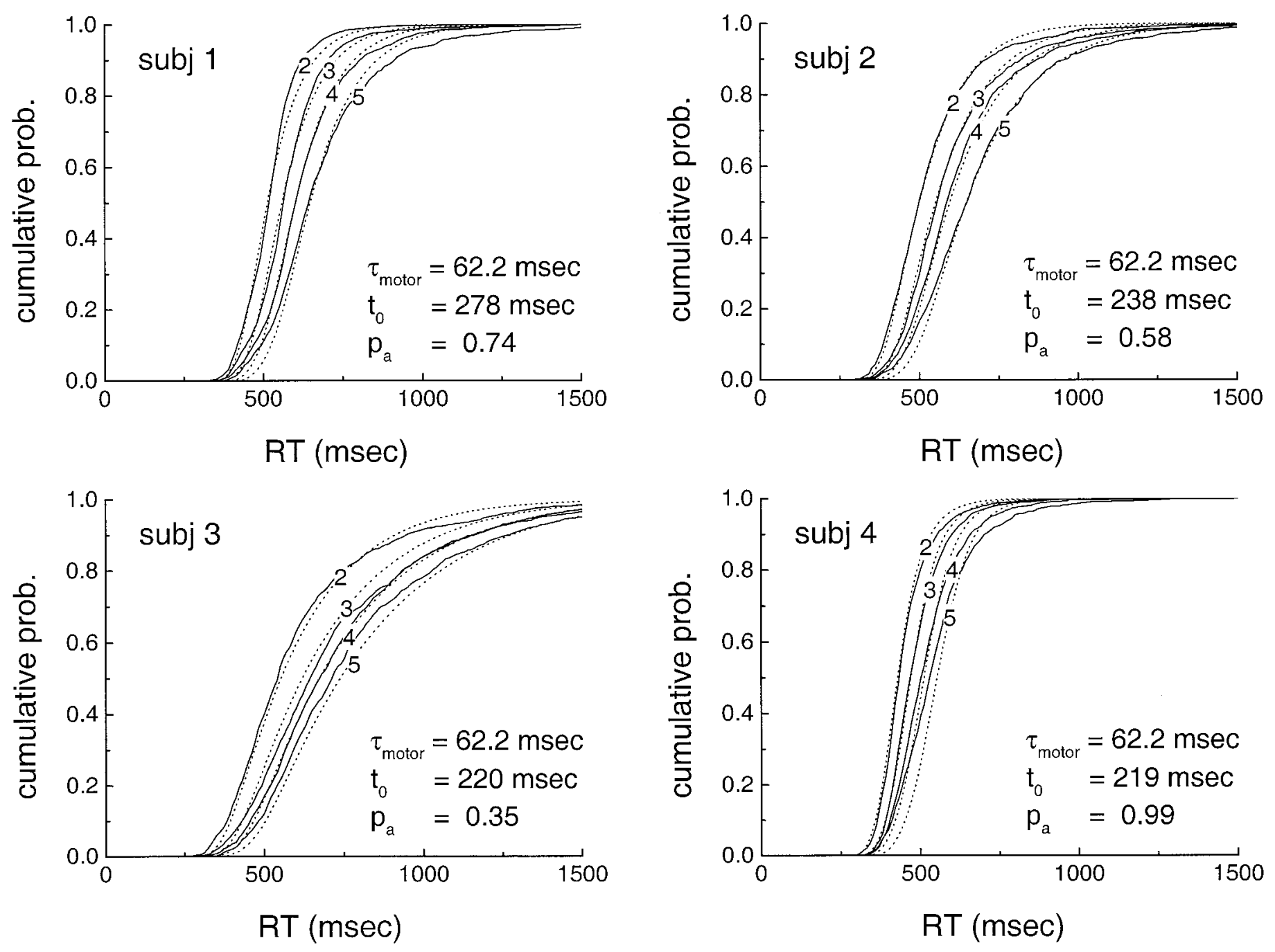

Figure 3. Adapting theta model (dashed lines) fitted to the cumulative RT distribution for both positive and negative probes (solid lines) measured by Ashby et al. (1993). By applying a least mean square method the cumulative distributions (Eq. 18) were fitted. The four values of $T_{\theta}$ are kept constant across subjects and experiments. For simplicity, $\tau_{\text {motor }}$ is kept the same for all subjects. $p_{\mathrm{a}}$ and $t_{0}$ are allowed to vary individually. Fitted values for $T_{\theta}(\mathrm{S}=$ 2, 3, 4, and 5) are 96, 119, 135, and $158 \mathrm{msec}$, respectively.

The probe is then serially compared with the items in the buffer (scanning) as they are activated $\left(t_{\text {scan }}\right)$. Finally, at the next trough the answering process may be initiated as a motor process of duration $t_{\text {motor }}$. In the simplest model, oscillations would be unaffected by presentation of the probe, and theta frequency would be independent of S. This last assumption means that even if only two memories are held, there are five "empty" gamma cycles. In this simple form the model cannot account for the increase in RT with S, because none of the times depends on S. Alternatively scanning might be initiated at the trough of a theta cycle and the motor response delivered at the end of the last active gamma cycle (not at the trough). In this model $t_{\text {wait }}$ does not depend on $\mathrm{S}$ but $t_{\text {scan }}$ does. This model can correctly reproduce the increase in RT with $\mathrm{S}$, because $t_{\text {scan }}$ depends on $\mathrm{S}$. However, it cannot account for the increase in variance with $S$, because the only term that depends on $\mathrm{S}$ is $t_{\text {scan }}$, which equals one theta cycle, and this by definition has no variance. These examples illustrate how models can fail to account for the detailed data. The next sections describe two models that can account for the data. The mathematical derivations are given in Appendix.

\section{Model I: theta frequency depends on the number of memories being stored (adapting theta model)}

Figure $4 A$ describes a model that successfully accounts for the RT data. The theta period increases with the number of items (S); i.e., the theta frequency decreases with S. Thus, a key feature of this model is that there are no "empty" gamma cycles even if the number of stored items is less than seven. When an additional item is added to STM, the theta period increases so there is an additional gamma cycle (up to a limit of seven for digits). Thus a theta period $\left(T_{\theta}\right)$ depends on the number of gamma periods $\left(T_{\gamma}\right)$ per theta cycle:

$$
T_{\theta}=T_{\gamma}(\mathrm{S}-1)+d .
$$

Here $d$ is a "dead time" denoting the part of the trough of the theta oscillations where no memories are active (Fig. 1). Such "theta adaptation" is plausible given the observed frequency variability of brain theta oscillations: correlations between memory tasks and oscillations have been observed in the 4-7 Hz band (Gevins et al., 1997; Klimesch et al., 1997; Sarnthein et al., 1998) 
A

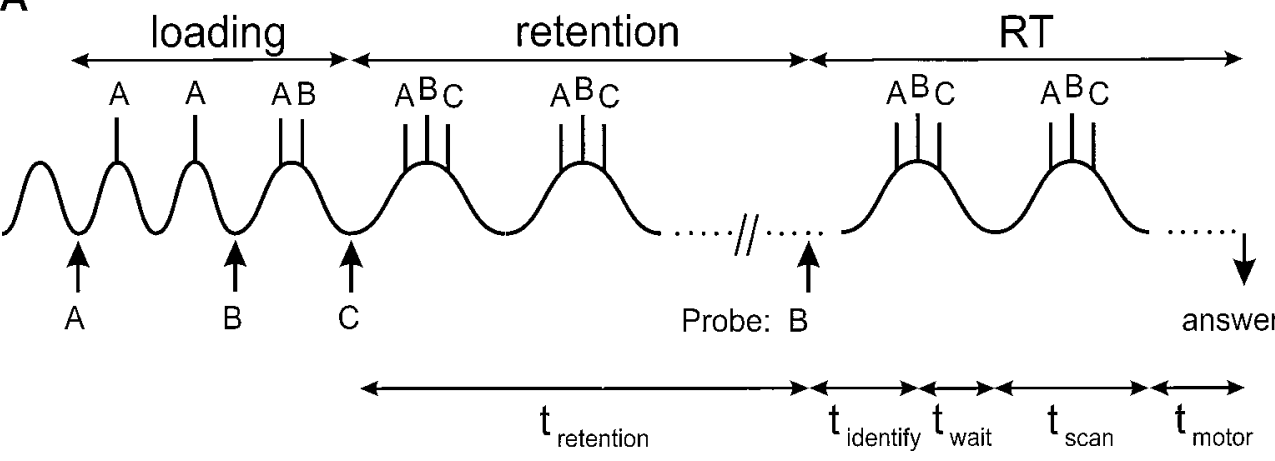

B
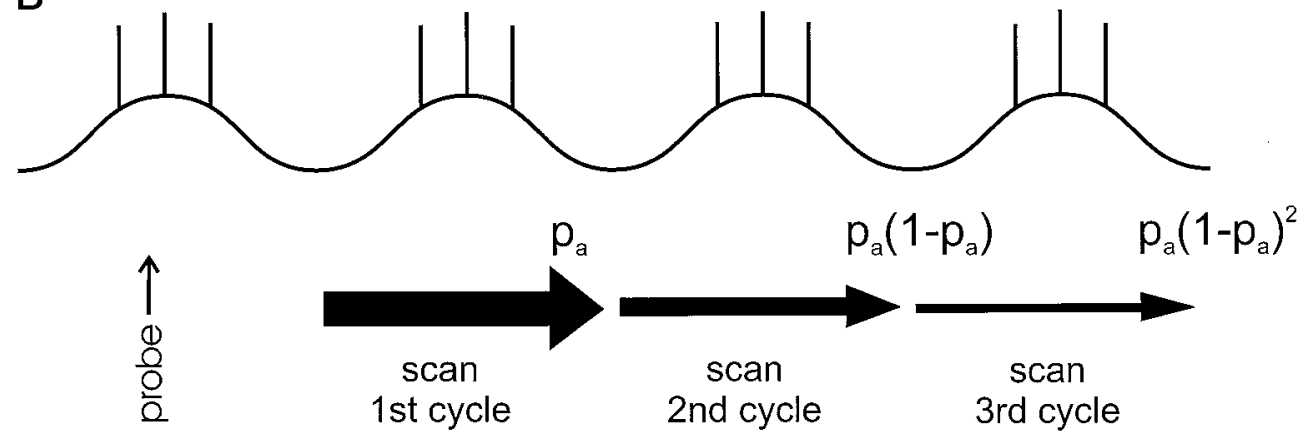

Figure 4. Diagrams indicating definition of relevant times and the concept of adapting theta. $A$, Three items $(A-C)$ are loaded; the theta period increases by one gamma period as each additional item is loaded (Eq 1). After loading, there is a delay period $\left(t_{\text {retention }}\right)$ in which the items are maintained by activity-dependent intrinsic properties of the neurons coding these items (Lisman and Idiart 1995; HajDahmane and Andrade, 1997). After probe presentation the items can be scanned, i.e., compared with the probe as they are activated. Scanning must wait until a trough of the theta cycle is reached, giving rise to a wait time $\left(t_{\text {wait }}\right)$. After scanning the motor response is initiated at the first possible trough, contributing to the time, $t_{\text {motor }} B$, There is a high probability, $p_{\mathrm{a}}$, that the answer will be initiated after the first complete scan. If not, scanning will be repeated and the response initiated at the end of the second scan with probability $p_{\mathrm{a}}\left(1-p_{\mathrm{a}}\right)$. The figure shows the probability of response after $n$ scans (a geometric distribution; Eq. 11). This skipping process is responsible for the increase in skewness (third moment) of the RT distribution with S.

and also at higher frequencies (10-12 Hz) (Krause et al., 1996). Note that in contrast to the subsequent model, we assume here that the phase of theta is not reset by the probe. Because $T_{\theta}$ varies with $\mathrm{S}$, a wait time, $t_{\text {wait }}$, described by a uniform distribution (Eq. 8 ), is introduced. Thus, in this model both mean and variance increase with $\mathrm{S}$, in accord with the data.

The motor response is described by an exponential distribution with a constant offset (Eq. 3), contributing $t_{\text {motor }}$ to the average $\mathrm{RT}$. The motor response gives a constant contribution to the variance of the RT distribution independent of S.

These assumptions alone predict a symmetrical RT distribution, but the observed distribution is skewed and has a skewness that increases with S (Fig. 2). To account for these features, we assumed that the probability of giving an answer $\left(p_{\mathrm{a}}\right)$ at the end of a theta cycle is $<1$; if no answer is given, scanning is repeated (Fig. 4B). We term this "skipping." The long RTs produced by skipping will produce the skewness in the distribution. The longer the theta period, the longer the duration of a skip. Thus, this effect can account for the increase in skewness with S (it also adds to the mean and variance of the RT). From these assumptions the full RT distribution can be derived (Eq. 17), as well as the mean, variance, and skewness (Eqs. 13, 15, 16). These expressions can be fit to the experimental data by finding the best parameters $T_{\gamma}$, $d, p_{\mathrm{a}}, \tau_{\text {motor }}$, and $t_{0}$. The constant $t_{0}$ determines the offset of the cumulative distribution and is defined in Equation 7. The dotted lines in the graphs of Figure 2 are the best fit of this model and account well for how the average, variance, and skewness of the RT distribution increase with S. These data were provided to us by S. Sternberg (University of Pennsylvania).

The model can also be tested against the data of Ashby et al. (1993), which provides a complete RT distribution. In Figure 3 we have replotted the RT distributions from Ashby et al. (1993) as cumulative distributions. In fitting the data it was assumed for simplicity that all subjects have the same theta period $\left(T_{\theta}\right)$ for a given value of $\mathrm{S}$. The free parameters are $T_{\theta}(S)$, which depends on
$T_{\gamma}$ and $d$, and $\tau_{\text {motor }}, t_{0}$, and $p_{\mathrm{a}}$. The latter two are assumed to be unique for each subject. The derived cumulative distributions were fit to the data using a procedure that minimizes the least square error.

The cumulative distributions are well fit by the model for all values of $\mathrm{S}$ and for all subjects (Fig. 3). The derived values of $T_{\theta}$ as a function of $\mathrm{S}$ obtained from the fits are shown in Figure 5. Note that the increase in theta with $\mathrm{S}$ is linear, even though all the theta values were fit to individual values of $S$. As $S$ varies, the theta period varies from 96 to $158 \mathrm{msec}(5.8-12.3 \mathrm{~Hz})$. These numbers are in plausible agreement with observed values in humans, which show considerable variability [4-7 Hz (Klimesch et al., 1997), 5.9 Hz (Gevins et al., 1997), 4-7 Hz (Sarnthein et al., 1998), 10-12 Hz (Krause et al., 1996)]. The gamma period $\left(T_{\gamma}\right)$ is the slope of the increase in $T_{\theta}$ with $\mathrm{S}$, and the dead time $(d)$ is the intercept (Fig. 5). The frequency of gamma derived in this way is $49 \mathrm{~Hz}$, consistent with typical values of gamma frequency (Ga-

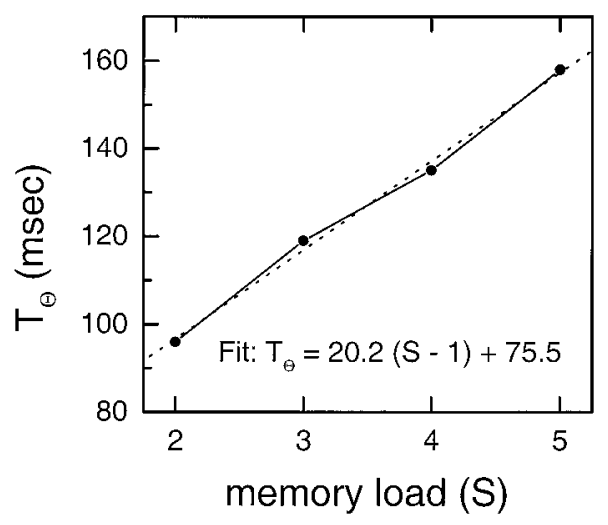

Figure 5. Theta values obtained from the fits in Figure 3 increase linearly with S. The slope and intercept of the best fit (dashed line) determine the gamma frequency and dead time: $T_{\gamma}=20.2 \mathrm{msec}$; and $d=75.5 \mathrm{msec}$. 


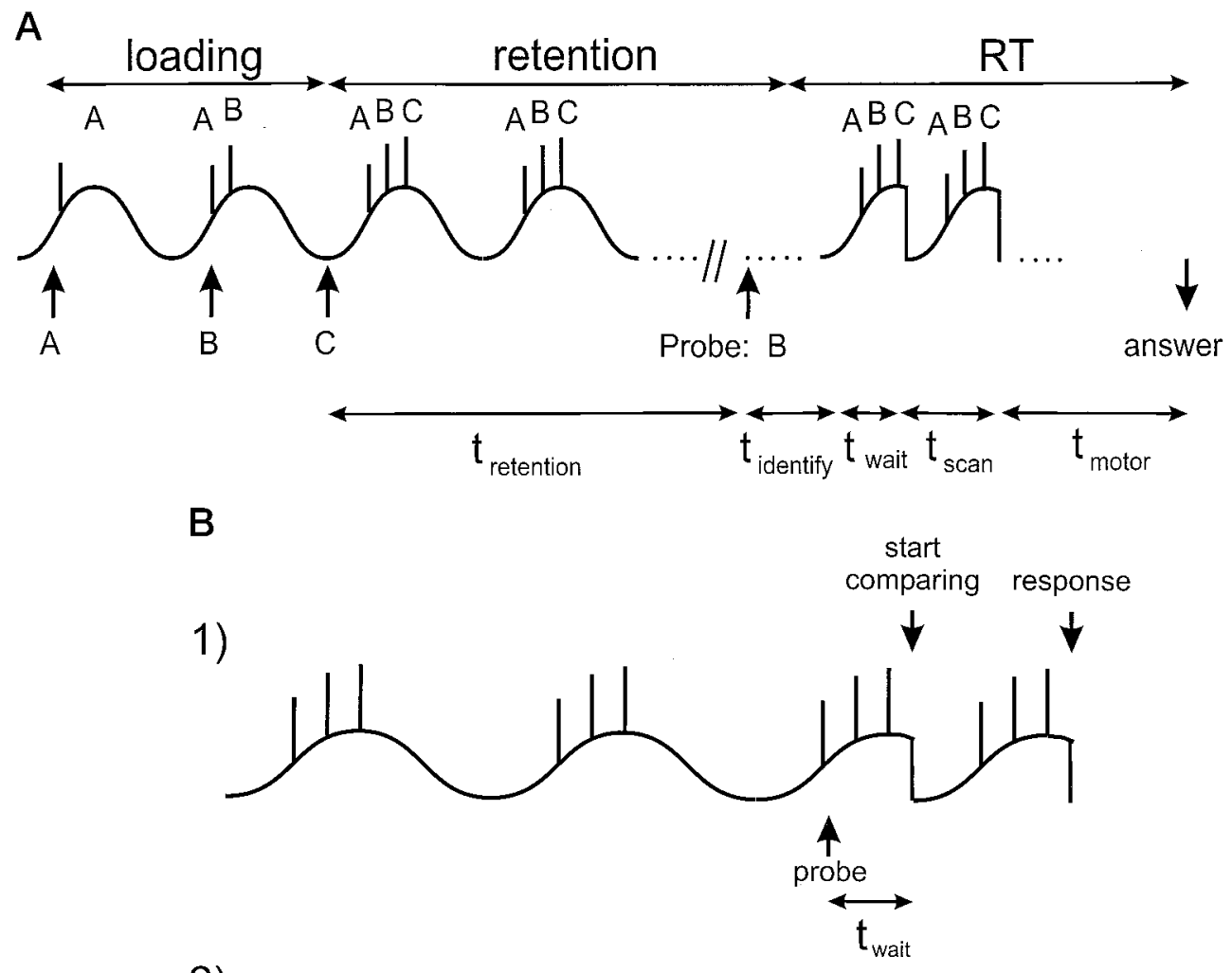

Figure 6. Reset model for RT in the Sternberg task. $A$, As for the adapting theta model, memories are loaded into the STM and kept active on subsequent theta cycles. In the reset model the theta frequency is independent of the memory load. After the probe arrives the theta oscillation is reset before scanning begins. $B$, Wait time $\left(t_{\text {wait }}\right)$ of the reset model. If the probe arrives when memories are actively read out, reset is delayed until after the last item (1). Otherwise, reset occurs immediately (2). This principle introduces a wait time described by Equation 23. The wait time contributes to the increase in variance with $\mathrm{S}$ but also the mean RT. $C$, As in the adapting theta model, skipping occurs if an answer is not available after the first scan trough. In this case the slow-wave oscillation is reset again, and another scan is initiated. This process is described by Equation 25 and accounts for the increase in skewness with S.

2)


lambos et al., 1981; Pantev et al., 1991; Joliot et al., 1994; Singer and Gray, 1995; Tallon-Baudry et al., 1997, 1998). The dead time $d$ is 75.5 msec.

Note that we obtained all the fits keeping the theta period at each $S$ (and hence the gamma period) the same for all the subjects. Hence individual differences were accounted for by variation in $t_{0}$ and $p_{\mathrm{a}}$, both of which are assumed to be independent of $\mathrm{S}$. The variations in $t_{0}$ might simply reflect individual differences in the rapidity of motor processes. Individual differences in attentiveness might explain variations in $p_{\mathrm{a}}$.

\section{Model II: reset of the slow-wave oscillation}

We next asked whether it was possible to find a satisfactory model in which theta adaptation did not occur. This implies that when the buffer has fewer items than seven there will be empty "slots" (Fig. 6A). As stated previously, simple models without theta adaptation do not work. We have considered the possibility that the phase of ongoing theta oscillations is "reset" each time a memory set is scanned. There is evidence for the reset of the theta oscillations in some brain regions (Berger et al., 1983; Rahn and Basar 1993a,b; Brankack et al., 1996; Brandt 1997). We 



Figure 7. Reset model (broken lines) fitted to the moments (mean, variance, and skewness) of the RT distributions (solid lines) from the data provided by Sternberg. The best fit to Equations 27, 29, and 30 resulted in the parameters $T_{\theta}=143 \mathrm{msec} ; p_{\mathrm{a}}=0.78 ; t_{0}=300 \mathrm{msec}$; and $\tau_{\text {motor }}=70$ msec. $d$ was fixed to $15.0 \mathrm{msec}$.

assume that the reset is controlled by the external mechanism driving the network at the theta rhythm. We have further assumed that the reset does not occur during the part of the theta cycle when the memories are actively being read out, because this would distort order information. If the probe identification is completed at a time when memories are being reactivated at gamma frequency, reset has to wait $\left(t_{\text {wait }}\right)$ until after activation of the last item (Fig. $6 B, 1)$. Otherwise the reset occurs immediately (Fig. 6B, 2).

After reset, scanning occurs from the trough to the end of the list (Fig. 6A). Thus, both $t_{\text {wait }}$ and $t_{\text {scan }}$ depend on $\mathrm{S}$. This gives rise to an increase in RT with $\mathrm{S}$, which is approximately linear. It further follows that the wait time (Fig. 6B) increases with S (Eq. 24). The random variation in $t_{\text {wait }}$ accounts for the major part of the increase in variance of RT with S. As in the previous model, a skipping process is assumed to account for the skewness of the distribution. The probability of the answer being given after the first scan is $p_{\mathrm{a}}$ (Fig. 6C). If for some reason the answer is not given, another scan is initiated after the theta oscillation has been reset. This produces the increasing asymmetry of the RT distribution with S (Eq. 25). The complete expressions for the mean, variance, and asymmetry (third central moment) are given by Equations 27, 29, and 30, with the free parameters $\tau_{\text {motor }}, t_{0}, p_{\mathrm{a}}$, $T_{\gamma}$, and $d$. The full RT distribution is given by Equation 31. Note that the final increase in RT with $\mathrm{S}$ has a second-order term (Eq. 28) because of the distribution of the wait time. However, for realistic parameter values, the increase in $\mathrm{RT}$ with $\mathrm{S}$ is approximately linear.

The three expressions fit quite well to the data of Sternberg
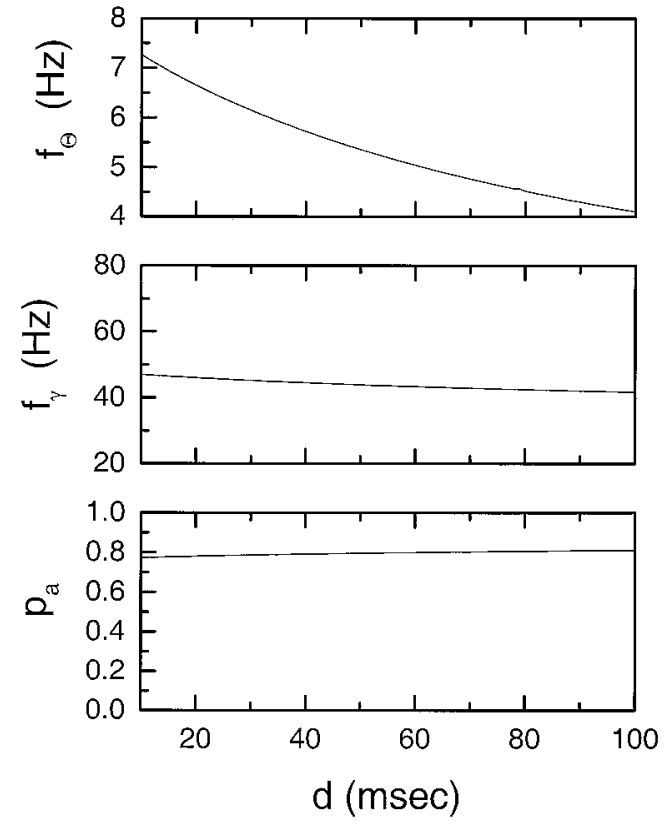

Figure 8. How varying $d$ influences the parameters. $d$ was fixed in the interval from 10 to $100 \mathrm{msec}$, and the other parameters were fitted to the Sternberg data in Figure 7. The fits remained well within the experimental SEs. Note that only $f_{\theta}$ varies strongly on $d$.

(Fig. 7). The fit is, however, not unique; we can vary the dead time, $d$ (see Eq. 20), in the interval from 10 to $100 \mathrm{msec}$ and still obtain fits to the Sternberg data within the SEs. The resulting values for $f_{\theta}, f_{\gamma}$, and $p_{\mathrm{a}}$ are shown in Figure 8 . Note that $p_{\mathrm{a}}$ and $f_{\gamma}$ do not depend significantly on $d$, whereas $f_{\theta}$ does. The dead time mainly adds to the theta period, but because scanning occurs from the trough to the end of the list, this does not change the retrieval properties of the model significantly.

As for the previous model, we have derived the expressions for the cumulative RT distributions to fit the data of Ashby et al. (1993) (Fig. 9). In this case $T_{\gamma}$ and $\tau_{\text {motor }}$ are kept the same for the four subjects, and $t_{0}$ and $p_{\mathrm{a}}$ are unique for each subject. $d$ was fixed to $15 \mathrm{msec}$ and $\mathrm{S}_{\text {span }}=7$.

We conclude that the reset model accounts for the RT data just as well as the adapting theta model. We are therefore not able to distinguish between the two models on the basis of the psychophysical data only. As will be discussed later, electrophysiological recordings on subjects performing the Sternberg task could be used to distinguish the two models.

\section{Accounting for serial position effect}

A major objection to serial scanning has been the existence of serial position effects: RT is systematically longer for early list items than late items (Fig. 10, first panel). Superficially, this is inconsistent with a scanning process in which each item is handled similarly. An important hint about the basis of this effect is that it disappears if the retention interval between list presentation and the probe is $>1.5 \mathrm{sec}$ (Fig. 10). This suggests that the serial position effect is not fundamental to scanning itself. For positive items, the probe is a repetition of a just-presented item, and we propose that the serial position effect is a consequence of short-term repetition priming (Bertelson and Renkin, 1966; Posner and Keele, 1967; Smith et al., 1973; McKone 1995); the time needed to identify the probe $\left(t_{\text {identify }}\right)$ is decreased if the item has been presented in the last few seconds. We have assumed that this 

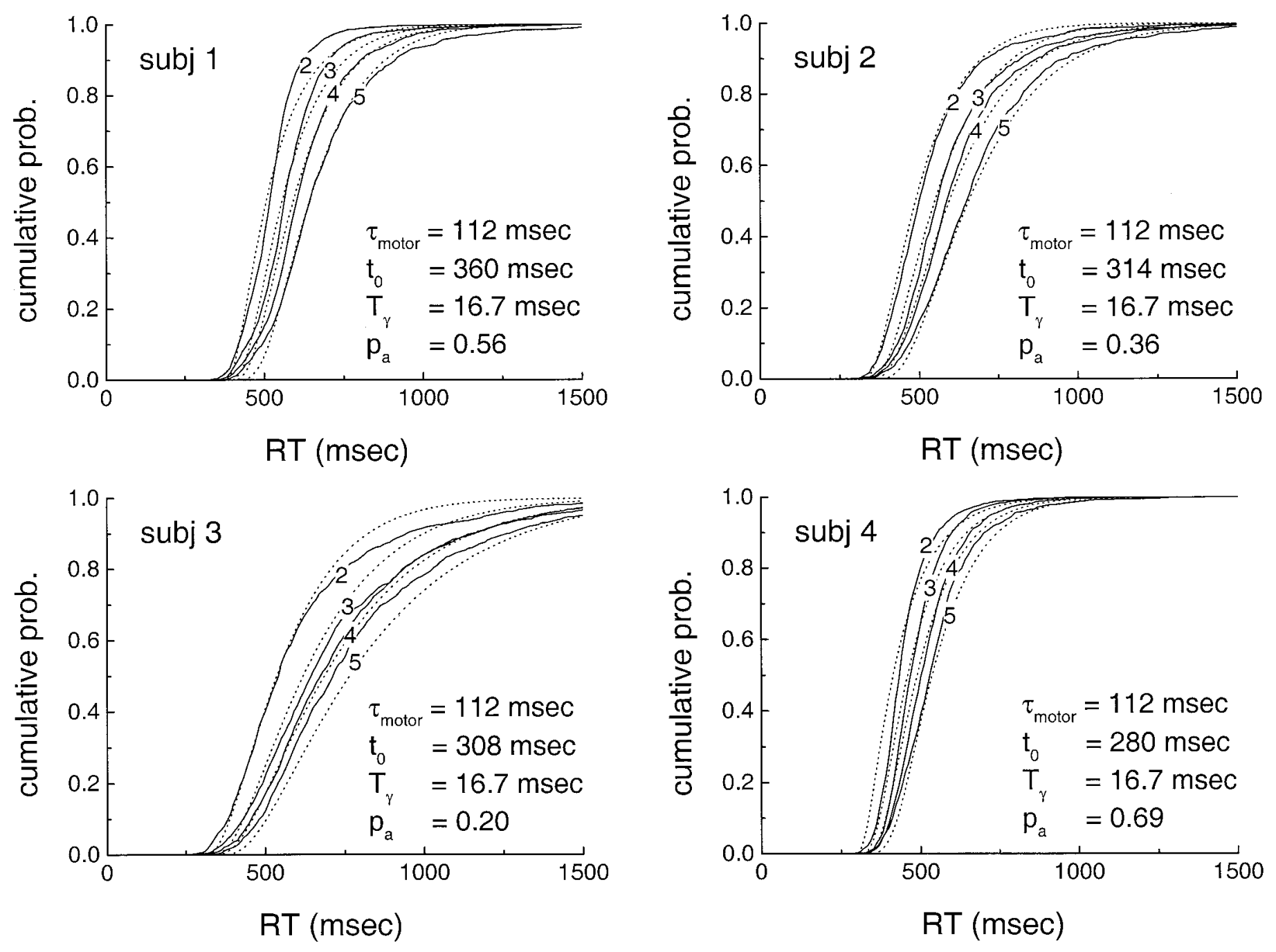

Figure 9. Reset model (dashed lines; Eq. 31) fitted to the cumulative RT distribution (solid lines) provided by Ashby et al. (1993). $p_{\mathrm{a}}$ and $t_{0}$ varied in between subjects, whereas $\tau_{\text {motor }}$ and $T_{\gamma}$ were the same for all subjects. The dead time was fixed to $d=15.0 \mathrm{msec}$.

priming decays exponentially ( $\tau_{\text {prime }}$; Eq. 33 , Fig. 11$)$. The time between the presentation of a memory item and the probe is a function of both the presentation rate, $f_{\text {rate }}$, and the retention interval, $t_{\text {retention }}$. The average time to identify the probe $\left(t_{\text {identify }}\right)$ is determined by Equation 35. Figure 10 shows that with a value of $\tau_{\text {prime }}=1.2 \mathrm{sec}$, this model nicely accounts for the serial position effects for various retention times.

Some experiments show that the average response to positive probes is slightly faster than for negative probes (Burrows and Okada, 1971; Corballis et al., 1972). This feature can be explained by repetition priming as well: only positive probes are primed and hence have a faster RT. Figure 12 shows how the adapting theta model captures this effect when a $500 \mathrm{msec}$ retention interval is used. The RT for positive items is $\sim 60-70 \mathrm{msec}$ faster than for negative items. From Figure 12 it can also be seen that the model predicts that the slope for positive items is $50 \%$ higher than the slope for negative items. According to our model this is a direct consequence of the priming effect resulting in faster RTs for positive items late is the list than items earlier in the list. This prediction is consistent with some experimental data in which fast presentation rates and short retention intervals were applied. Corballis et al. (1972) found that positive probes had $49 \%$ higher slopes than negative probes. Burrows and Okada (1971) found the value to be $18 \%$. Many factors can contribute to the differences in the ratios, such as presentation rate and training of the subjects.

\section{DISCUSSION}

\section{Two oscillatory buffer models explain the RT distributions}

We have found two models of a multiplexing buffer that can account quantitatively for the RT distributions of the Sternberg paradigm. Retrieval in these models is based on exhaustive serial search. The models fit both the moments of the RT distributions measured by Sternberg (1964) and the actual distributions measured by Ashby et al. (1993). They can account for the changes in these distributions with memory load and can be applied to different subjects without having to assume differences in fundamental oscillatory frequencies. The two models differ with regard to theta adaptation and phase reset. In the first model (adapting theta) the theta period varies with the number of items in STM, but there is no reset of the oscillations when the probe arrives. In the second model, the theta period is constant, but the oscillation is phase-reset by the probe. We later discuss how these assumptions can be tested. 




Figure 10. Data from Forrin and Cunningham (1973), showing that short-term repetition priming can explain serial position effects. The shorter the delay between presentation of list items and the probe $\left(t_{\text {retention }}\right)$, the faster the RT (recency), as shown by the solid lines. By modeling the priming by a simple exponential (Fig. 11), recency is fit reasonably well (broken lines). Best fit (least mean square): $\tau_{\text {prime }}$ $=1181 \mathrm{msec}$ and $\alpha=250 \mathrm{msec}$. Response times are plotted relative to serial position

Table 1 summarizes the fitted values for the two models. With respect to the gamma frequency the two models give values in the same range $(46-59 \mathrm{~Hz})$. These values are in good agreement with experimentally observed data (Galambos et al., 1981; Pantev et al., 1991; Joliot et al., 1994; Singer and Gray, 1995; Tallon-Baudry et al., 1997, 1998). In the adapting theta model, slow-wave frequency depends on $\mathrm{S}$ and varies from 6 to $10 \mathrm{~Hz}$, i.e., covering both the defined theta and alpha bands. Note that both the Sternberg and Ashby data sets give rise to very similar values. The reset model does not strongly constrain the value of the theta frequency and allows frequencies in the interval from 4 to $7 \mathrm{~Hz}$.

A key feature of the data that determines the value of gamma frequency is the systematic changes in the rising edge of the cumulative response time distribution with increasing S (Fig. 3). This is the closest psychophysics comes to direct detection of gamma frequency. However, an important conclusion of our work is that deriving gamma from the data requires a full model. We originally thought that the Sternberg slope (average increase in reaction time per item) might directly correspond to the gamma period (Lisman and Idiart, 1995). However, the models presented here show the correspondence is only approximate. This is because slope measurements are based on average RT, but this average is determined not only by $T_{\gamma}$ but also the wait time and skipping (Eqs. 13 and 27). Consequently, the slope is $\sim 1.5-2$ higher than the gamma period.

The rising edge of the cumulative response distributions of a

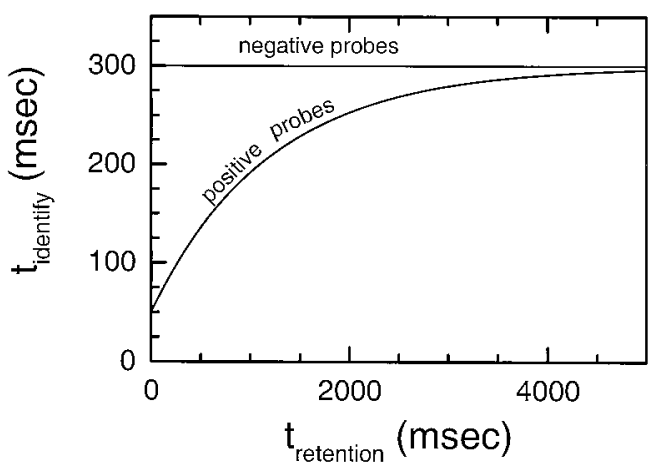

Figure 11. Repetition priming is modeled as a single exponential (Eq. 33). A positive probe is primed by the previous occurrence of the similar item during presentation. This reduces the time to identify the probe. Negative probes are not primed. task related to the Sternberg paradigm, the speed-accuracy tradeoff (SAT) (McElree and Dosher, 1989), does not have a latency depending on S, whereas the Sternberg task does (Fig. 3). The SAT data have been taken as an argument against serial scanning. However, the SAT methodology is different from conventional recognition memory paradigms in ways we believe limit the conclusions that can be drawn. In this test, the subject is urged to respond at some fixed time after the presentation of the test item by a signal to respond. The presence of this signal and its processing introduce processing problems not present in the Sternberg paradigm. Second, although the signal to respond is given at a precise time, the subsequent time to respond is not precise. This could be enforced by only considering responses given with a precise latency but is not. Because of this ambiguity, the method may not be able to resolve small relevant delays on the order of 30 msec.

Several other models have been proposed that can account for the main features of the RT distribution and the serial position effects. Cavanagh (1976) has investigated trace strength models and holographic models, which both accounted for the increase in RT with S and serial position effects of the Sternberg paradigm. Ratcliff (1978) and Hockley and Murdock (1987) suggested deci-

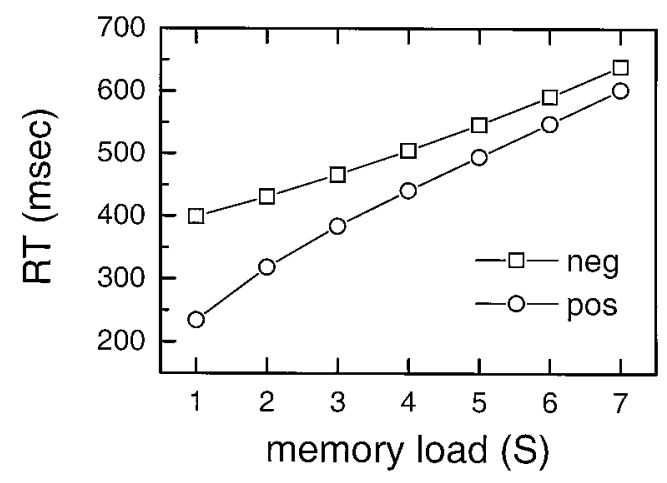

Figure 12. Repetition priming explains why the RT for positive items is faster than for negative items (which lack priming). Also, the slope of the RT curve for positive items should be slightly steeper than for negative items. The graph shows the mean RT calculated using Equations 21 and 36 for $t_{0}$. The parameters from the fit in Figure 7 were used, except $t_{0}=$ $70 \mathrm{msec}$. For the priming mechanism we used $\tau=1200 \mathrm{msec}$ and $\alpha=250$ msec. The presentation rate was $f_{\text {rate }}=1 / 1200 \mathrm{msec}^{-1}$; the probe delay was $t_{\text {probe }}=500 \mathrm{msec}$. 


\begin{tabular}{|c|c|c|c|c|c|c|}
\hline Model & Data & $\begin{array}{l}f_{\gamma} \\
(\mathrm{Hz})\end{array}$ & $\begin{array}{l}f_{\theta} \\
(\mathrm{Hz}) \\
\mathrm{S}=2,3,4,5\end{array}$ & $p_{\mathrm{a}}$ & $\begin{array}{l}d \\
(\mathrm{msec})\end{array}$ & $\begin{array}{l}\tau_{\text {motor }} \\
(\mathrm{msec})\end{array}$ \\
\hline \multirow[t]{8}{*}{$\begin{array}{c}\text { Adapting } \\
\text { theta }\end{array}$} & $\begin{array}{c}\text { Sternberg, } \\
1966\end{array}$ & 46 & 9.8 & 0.88 & 80.1 & 57.0 \\
\hline & & & 8.1 & & & \\
\hline & & & 6.8 & & & \\
\hline & & & 5.9 & & & \\
\hline & $\begin{array}{l}\text { Ashby et } \\
\quad \text { al., } 1993^{a}\end{array}$ & 50 & 10.4 & 0.67 & 75.5 & 62.2 \\
\hline & & & 9.4 & & & \\
\hline & & & 7.4 & & & \\
\hline & & & 6.3 & & & \\
\hline \multirow[t]{3}{*}{$\begin{array}{l}\text { Theta } \\
\text { reset }\end{array}$} & $\begin{array}{c}\text { Sternberg, } \\
1966\end{array}$ & 46 & $6.9^{b}$ & 0.75 & 15.0 & 70.0 \\
\hline & Ashby et & & & & & \\
\hline & al., $1993^{a}$ & 59 & $8.7^{b}$ & 0.45 & 15.0 & 112 \\
\hline
\end{tabular}

${ }^{a}$ Values averaged over four subjects.

${ }^{b}$ Values valid for dead time fixed to $d=15 \mathrm{msec}$.

sion type models, which also successfully accounted for the RT distributions and serial position effects. However, none of these theories has been implemented in a physiologically realistic way.

\section{Possible rationale for exhaustive search}

Sternberg concluded that memory search was exhaustive because RT depended similarly on $\mathrm{S}$ for positive probes as for negative probes (for which search is necessarily exhaustive). Because it would seem more efficient for the motor response to begin as soon as a positive match occurred (i.e., nonexhaustive search), some have argued that exhaustive search is implausible. Our oscillatory model, however, provides a plausible explanation for exhaustive search. All that needs to be assumed is that initiation of the motor response can only occur at the trough of the theta cycle. There are several examples in the literature of the importance of theta phase for information processing (Pavlides et al., 1988; O'Keefe and Recce, 1993; Nicolelis et al., 1995; Huerta and Lisman, 1996).

\section{Physiological tests for distinguishing between the models}

The adapting theta model can be tested by analyzing EEG, magnetoencephalography (MEG) and/or intracranial recordings on subjects performing the Sternberg task. Results from imaging studies suggest that prefrontal and parietal cortex are involved in the active maintenance of STM (Shallice and Vallar, 1990; Cohen et al., 1997a,b; Jonides et al., 1998). We therefore expect to observe the predicted changes in theta and gamma oscillations in these areas. If the model is correct, the slow-wave oscillation during the retention period should systematically decrease in frequency with higher memory loads. Oscillations in the theta band have been identified in humans performing STM tasks using both EEG and MEG recordings (Gundel and Wilson, 1992; Mecklinger et al., 1992; Gevins et al., 1997; Klimesch et al., 1997; Sarnthein et al., 1998). The band in which theta oscillations have been observed is sufficiently broad to allow the predicted adaptation with memory loads, but whether these variations are related to memory load remains to the tested.

The two models make different predictions about the effect of $\mathrm{S}$ on overall neuronal firing and thus on the signals detected by brain-imaging methods. In the reset model, the fraction of the

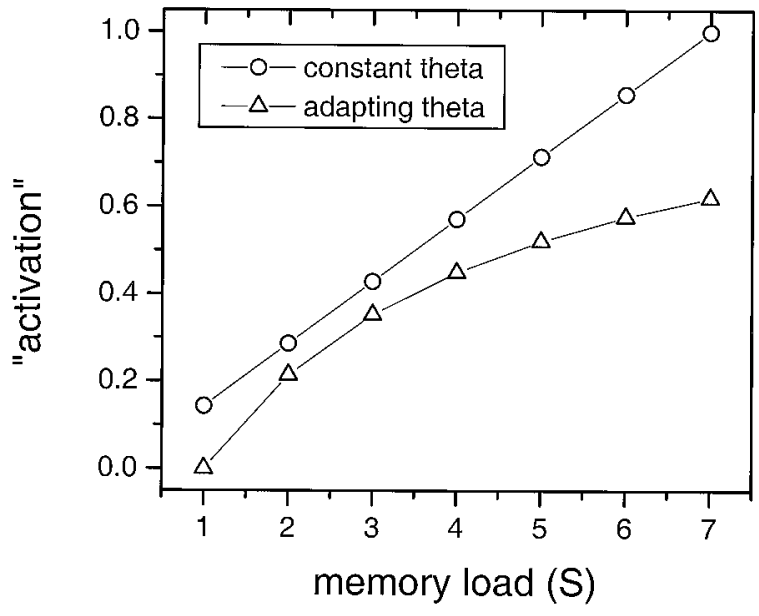

Figure 13. Both of the proposed models predict an increase in overall neuronal activity with $\mathrm{S}$. The constant theta model predicts a linear increase attributable to temporal summation of neurons participating in the memory representation. In the adapting theta model, a sublinear increase is expected: activation $=\left[\mathrm{T}_{\gamma}(\mathrm{S}-1)\right] /\left[\mathrm{T}_{\gamma}(\mathrm{S}-1)+\mathrm{d}\right]$.

theta period being occupied by active memory representations increases with $\mathrm{S}$. Consequently, the number of activated neurons per theta cycle will increase linearly with S (Fig. 13). In contrast, the adapting theta model predicts a sublinear increase with $\mathrm{S}$ (Fig. 13). These predictions may be relevant in interpreting the data of Cohen et al. (1997a,b), which showed that memory load (S) increases functional MRI signals in prefrontal cortex, parietal areas, and Broca's area.

Examination of the load dependence of EEG amplitude may provide another way of distinguishing the models. The factors controlling the amplitude of EEG are unclear, but it seems plausible that amplitude would vary with the number of neurons that fire within an oscillatory cycle. Hence one might expect the theta amplitude to increases with S. A study by Gevins et al. (1997) points in this direction: in an $n$-back task a higher power of frontal-midline theta was observed for $n=3$ than for $n=1$.

A critical distinction between the adapting theta and reset models may be made by studying the reset of the slow-wave oscillations after the arrival of the probe. When $\mathrm{S}$ is small, there will be a fairly large period during which there will be no cells firing. If the end of item identification occurs during this period, the phase of oscillation will be reset, and the resulting waveforms will be synchronized with the probe onset. Although there will also be a less synchronized component (if identification occurs during firing), the synchronous component will be identifiable as an oscillation in the averaged EEG, the evoked potential. Such a signal would not be generated if the adapting theta model is correct. Several groups (Rahn and Basar, 1993a,b; Brandt, 1997) have reported components in the $\alpha$ range of averaged EEG traces after simple stimuli, suggesting reset of the slow-wave oscillations.

\section{Short-term repetition priming can account for serial position effects}

Serial position effects have been used as an argument against serial scanning. However, if the time for encoding of the probe can be primed by previous exposure, our model can account quantitatively for the serial position effect and retain the idea of serial scanning. The decay of priming with $\tau=1.2 \mathrm{sec}$ explains why the serial position effect becomes smaller for longer retention intervals. Furthermore, priming explains why reaction times are 
shorter for positive than negative probes, and why the RT slope (increase in RT with S) is slightly steeper for positive than for negative probes (Fig. 12). Short-term repetition priming with a duration of a few seconds has been observed in other psychophysical tests not necessarily related to memory paradigms (Bertelson and Renkin, 1966; Posner and Keele, 1967; Smith et al., 1973; McKone, 1995). Monsell (1978) showed that if an item has been used as a negative probe in the previous trial, the response time increases in the subsequent trial if the same item is used as a positive probe. This finding has been used to argue against priming as an explanation for the serial position effect. However, this effect occurs over $10-15 \mathrm{sec}$, whereas repetition priming decays in a few seconds; hence different mechanisms may be involved.

Our proposal that priming of the encoding of the probe accounts for serial position effects (Fig. 10) leads to further predictions within the context of the reset model. A reset of the theta cycle follows the arrival of the probe. This reset will occur earlier for probes matching items late in the list compared with items early in the list. It should thus be interesting to look for the effect of serial position on the latency of evoked potentials.

In conclusion, we have found that a physiologically realistic model of serial memory search can account quantitatively for a large body of the data on STM. Furthermore, we have been able to counter some of the major objections to exhaustive serial search. Although it is certainly true that brain computations can occur in parallel, our results suggest that there may also be an important role for serial processing.

\section{APPENDIX}

\section{Derivation of RT distribution for model I}

In the first model the theta period $\left(T_{\theta}\right)$ depends on the memory load $(\mathrm{S})$ and dead time $(d)$ according to Equation 1.

The average reaction time (RT) is the sum of the following terms (see Fig. 4A):

$$
\mathrm{RT}=t_{\text {identify }}+t_{\text {wait }}+t_{\text {scan }}+t_{\text {skip }}+t_{\text {motor }} .
$$

The motor response is simply described by an exponential distribution:

$$
p_{\text {motor }}(t)=c_{\text {motor }}+\frac{1}{\tau_{\text {motor }}} e^{-t / \tau_{\text {motor }}}, t>0,
$$

where the contribution to RT is the mean of this distribution:

$$
t_{\text {motor }}=c_{\text {motor }}+\tau_{\text {motor }}
$$

The time from presentation of a memory item until it is available for insertion into the oscillatory buffer is termed $t_{\text {identify. This }}$ variable is as a first-assumption constant:

$$
t_{\text {identify }}=c_{\text {identify }} .
$$

For convenience we define

$$
t_{\text {identify }}+t_{\text {motor }}=t_{0}+\tau_{\text {motor }},
$$

where

$$
t_{0}=c_{\text {motor }}+c_{\text {identify }} .
$$

The probe can become available anywhere within a theta cycle and a wait time is introduced, described by a uniform distribution:

$$
p_{\text {wait }}(t)=1 / T_{\theta}, 0<t<T_{\theta}
$$

with the mean

$$
t_{\text {wait }}=T_{\theta} / 2 \text {. }
$$

The memories in the buffer are scanned from trough to trough:

$$
t_{\text {scan }}=T_{\theta},
$$

where $T_{\theta}$ is defined by Equation 1 . The probability that an answer is available after scanning trough the first possible theta cycle is $p_{\mathrm{a}}$. In case an answer is not available, another scan has to be performed, and so forth. Hence the probability that a response is initiated in theta cycle $n$ is described by a geometric distribution:

$$
p_{\text {skip }}(t)=p_{\mathrm{a}}\left(1-p_{\mathrm{a}}\right)^{n}, \text { where } n=\left[t / T_{\theta}\right], t>0 .
$$

This contributes to the mean of RT as

$$
t_{\text {skip }}=\left(\frac{1}{p_{\mathrm{a}}}-1\right) T_{\theta} .
$$

Finally, the mean of RT is obtained from the sum of Equations 6, 10, 9, and 12:

$$
\operatorname{Mean}\{\mathrm{RT}\}=t_{0}+\tau_{\text {motor }}+T_{\theta}\left(\frac{1}{2}+\frac{1}{p_{\mathrm{a}}}\right) .
$$

The slope of RT with S is given by

$$
\alpha=\left(\frac{1}{2}+\frac{1}{p_{\mathrm{a}}}\right) T_{\gamma} .
$$

The variance of the response time distribution is the sum of variances from the distributions in Equations 3, 8, and 11:

$$
\operatorname{Var}\{\mathrm{RT}\}=\tau_{\text {motor }}^{2}=\frac{\mathrm{T}_{\theta}^{2}}{12}+\frac{1-p_{\mathrm{a}}}{p_{\mathrm{a}}^{2}} T_{\theta^{\circ}}^{2}
$$

Only Equations 3 and 11 contribute to the third central moment measuring skewness:

$$
\operatorname{Trd}\{\mathrm{RT}\}=2 \tau_{\text {motor }}^{3}+\frac{(1-p)(2-p)}{p_{\mathrm{a}}^{3}} T_{\theta}^{3} .
$$

By convolving the distributions, Equations 3, 8, and 11, the full expression for the RT distribution is obtained:

$$
p_{\mathrm{RT}}(t)=p_{\text {motor }}(t)^{*} p_{\text {wait }}(t)^{*} p_{\text {skip }}(t) .
$$

The cumulative distributions is:

$$
P_{\mathrm{RT}}(t)=\int_{0}^{t} p_{\mathrm{RT}}\left(t^{\prime}\right) d t^{\prime} .
$$

In this paper we calculated the full distribution by numerical integration. Both the moments (Eqs. 13, 15, and 16) and the expressions for the cumulative distribution (Eq. 18) have been fitted to the psychophysical data. The parameters to determine are $\tau_{\text {motor }}, t_{0}, p_{\mathrm{a}}, T_{\gamma}$, and $d$. To fit the moments of the Sternberg data (1964), we first obtained the linear slopes (obtained by regression) of the mean, variance, and third central moments of both the data and the model. In the fitting algorithm the differences between the weighted slopes,

$$
f=\left(\alpha_{\text {mean }}^{\text {model }}-\alpha_{\text {mean }}^{\text {data }}\right)+\left(\alpha_{\text {var }}^{\text {model }}-\alpha_{\text {var }}^{\text {data }}\right)^{1 / 2}+\left(\alpha_{\text {trd }}^{\text {model }}-\alpha_{\text {trd }}^{\text {data }}\right)^{1 / 3},
$$


were minimized by adjusting $T_{\gamma}, d$, and $p_{\mathrm{a}}$. Then $\tau_{\text {motor }}$ and $t_{0}$ were adjusted to fit the intercepts of the moments. The cumulative distributions (18) were directly fitted to the data of Ashby et al. (1993).

\section{Derivative of RT distribution for model II}

In this model the period of the slow-wave oscillation $\left(T_{\theta}\right)$ is constant. It is derived from the gamma period ( $\left.T_{\text {gamma }}\right)$, dead time $(d)$, and the maximum memory span, which is set to $S_{\text {span }}=7$ :

$$
T_{\theta}=T_{\gamma}\left(S_{\mathrm{span}}-1\right)+d
$$

Again the average reaction time (RT) is the sum of the following terms:

$$
\mathrm{RT}=t_{\text {identify }}+t_{\text {wait }}+t_{\text {scan }}+t_{\text {skip }}+t_{\text {motor }} .
$$

Scanning occurs from the trough to the end of the list, so:

$$
t_{\text {scan }}=(S-1) T_{\gamma}+d .
$$

Reset of the theta oscillation after arrival of the probe is assumed. The reset, however, cannot occur when a set of memories are actively read out, because this will distort order information. This principle introduces a wait time determined by

$$
p_{\text {wait }}(t)=\left\{\begin{array}{cc}
\delta(t) T_{\theta} / t_{\text {scan }} & \text { if } t=0 \\
1 / T_{\theta} & \text { if } 0<t<t_{\text {scan }} \\
0 & \text { otherwise }
\end{array}\right.
$$

with the mean

$$
t_{\text {wait }}=\frac{1}{2} t_{\text {scan }}^{2} / T_{\theta} \text {. }
$$

$\delta(t)$ denotes the delta function. The argument for the skipping mechanism is the same as in Equation 11, with the additional assumption that the theta oscillation is reset after a skip:

$$
p_{\text {skip }}(t)=p_{\mathrm{a}}\left(1-p_{\mathrm{a}}\right)^{n}, \text { where } n=\left[t / t_{\text {scan }}\right], t>0 .
$$

This contributes to RT as

$$
t_{\text {skip }}=\left(\frac{1}{p_{\mathrm{a}}}-1\right) t_{\text {scan }}
$$

Finally, the mean of the response time distribution is obtained by Equations 6, 22, 24, and 26:

$$
\operatorname{Mean}\{\mathrm{RT}\}=t_{0}+\tau_{\text {motor }}+\frac{1}{2} \frac{t_{\text {scan }}^{2}}{T_{\theta}}+\left(\frac{1}{p_{\mathrm{a}}}-1\right) t_{\text {scan }} .
$$

The increase in RT with S takes the form

$$
\mathrm{RT}=T_{\gamma}\left(\frac{1}{p_{\mathrm{a}}}+\frac{d}{T_{\theta}}-\frac{T_{\gamma}}{T_{\theta}}\right) S+\frac{T_{\gamma}^{2}}{2 T_{\theta}} S^{2}+\text { constant. }
$$

This expression approximates a linear increase with $\mathrm{S}$ for reasonable values of $T_{\gamma}$ and $T_{\theta}$. The variance of the response time distribution is the sum of variances from Equations 3, 23, and 25:

$$
\operatorname{Var}\{\mathrm{RT}\}=\tau_{\text {motor }}^{2}+\frac{t_{\text {scan }}^{3}}{T_{\theta}}\left(\frac{1}{3}-\frac{1}{2} \frac{t_{\text {scan }}}{T_{\theta}} \frac{1}{4}\left(\frac{t_{\text {scan }}}{T_{\theta}}\right)^{2}\right)+\frac{\left(1-p_{\mathrm{a}}\right)}{p_{\mathrm{a}}^{2}} t_{\text {scan }}^{2} .
$$

The third central moment stems from the motor response (Eq. 3) and skipping (Eq. 25):

$$
\operatorname{Trd}\{\mathrm{RT}\}=2 \tau_{\text {motor }}^{3}+\frac{\left(1-p_{\mathrm{a}}\right)\left(2-p_{\mathrm{a}}\right)}{p_{\mathrm{a}}^{3}} t_{\text {scan }}^{3} .
$$

The parameters to determine are $\tau_{\text {motor }}, t_{0}, p_{\mathrm{a}}, d$, and $T_{\gamma}$. The fitting method is the same as for the adapting theta model.

As for the adapting theta model an expression for the full RT distribution can be obtained by convolving the individual distributions (Eqs. 3, 23, 25):

$$
p_{\mathrm{RT}}(t)=p_{\text {motor }}(t)^{*} p_{\text {wait }}(t)^{*} p_{\text {skip }}(t) .
$$

\section{Serial position}

The time to identify a positive probe is shortened (primed) by the recent introduction of the equivalent item during presentation of the list. The time between the presentation of item $i$ and the probe is given by:

$$
t_{\text {retention }, i}=\frac{1}{f_{\text {rate }}}(S-i)+t_{\text {probe }},
$$

where $f_{\text {rate }}$ is the presentation rate of the items, and $t_{\text {probe }}$ is the delay between presentation of the last items and arrival of the probe. We assume that the time it takes to identify an item is determined by an exponentially saturating function:

$$
t_{\text {prime }}(t)=\alpha\left(1-e^{-t / \tau_{\text {prime }}}\right),
$$

where $t$ denotes the time since previous presentation. Hence the time to identify item $i$ is:

$$
t_{\text {identif }, i}=c_{\text {prime }}+t_{\text {prime }}\left(\frac{1}{f_{\text {rate }}}(S-i)+t_{\text {probe }}\right) .
$$

The average identification time for a set of $\mathrm{S}$ items is then:

$t_{\text {identify }}=\frac{1}{S} \sum_{i=1}^{S} t_{\text {identify }, i}=c_{\text {prime }}+\frac{1}{S} \sum_{i=1}^{S} t_{\text {prime }}\left(\frac{1}{f_{\text {rate }}}(S-i)+t_{\text {probe }}\right)$.

Note that $t_{\text {identify }}=\alpha+c_{\text {prime }}$ for negative probes, and $t_{\text {retention }}$ $\gg \tau_{\text {prime }}$. When applying the priming mechanism to the models, Equation 6 is rewritten to:

$$
t_{\text {identify }}+t_{\text {motor }}=t_{0}+\tau_{\text {motor }}+\frac{1}{S} \sum_{i=1}^{S} t_{\text {prime }}\left(\frac{1}{f_{\text {rate }}}(S-i)+t_{\text {probe }}\right),
$$

where

$$
t_{0}=c_{\text {motor }}+c_{\text {prime }}
$$

\section{REFERENCES}

Ashby FG, Tein J, Balakrishnan JD (1993) Response time distributions in memory scanning. J Math Psychol 37:526-555.

Atkinson RC, Shiffrin RM (1968) Human memory: a proposed system and its control processes. In: The psychology of learning and motivation: advances in research and theory, Vol. 2, (Spence KW, ed). New York: Academic.

Berger TW, Rinaldi PC, Weisz DJ, Thompson RF (1983) Single-unit analysis of different hippocampal cell types during classical conditioning of rabbit nictitating membrane response. J Neurophysiol 50:1197-1219.

Bertelson P, Renkin A (1966) Reaction times to new versus repeated signals in a serial task as a function of response-signal time interval. Acta Psychol 25:132-136.

Bragin A, Jando G, Nadasdy Z, Hetke J, Wise K, Buzsaki G (1995) 
Gamma (40-100 Hz) oscillation in the hippocampus of the behaving rat. J Neurosci 15:47-60.

Brandt ME (1997) Visual and auditory evoked phase resetting of the alpha EEG. Int J Psychophysiol 26:285-298.

Brankack J, Seidenbecher T, Muller-Gartner HW (1996) Task-relevant late positive component in rats: is it related to hippocampal theta rhythm? Hippocampus 6:475-482.

Brener R (1940) An experimental investigation of memory span. J Exp Psychol 26:467-482.

Burrows D, Okada R (1971) Serial position effects in high-speed memory search. Percept Psychophys 10:305-308.

Cavanagh P (1976) Holographic and trace strength models of rehearsal effects in the item recognition task. Mem Cognit 4:186-199.

Clifton C, Birenbaum S (1970) Effects of serial position and delay of probe in memory scan task. J Exp Psych 86:69-76.

Cohen JD, Nystrom LE, Sabb FW, Braver TS, Noll DC (1997a) Tracking the dynamics of fMRI activation in humans under manipulations of duration and intensity of working memory processes. Soc Neurosci Abstr 23:1678.

Cohen JD, Perlstein WM, Braver TS, Nystrom LE, Noll DC, Jonides J, Smith EE (1997b) Temporal dynamics of brain activation during a working memory task. Nature 386:604-608.

Corballis MC, Kirby J, Miller A (1972) Access to elements of a memorized list. J Exp Psychol 94:185-190.

Forrin B, Cunningham K (1973) Recognition time and serial position of probed item in short-term memory. J Exp Psychol 99:272-279.

Fuster JM (1997) Network memory. Trends Neurosci 20:451-459.

Galambos R, Makeig S, Talmachoff PJ (1981) A 40-Hz auditory potential recorded from the human scalp. Proc Natl Acad Sci USA 78:2643-2647.

Gevins A, Smith ME, McEvoy L, Yu D (1997) High-resolution EEG mapping of cortical activation related to working memory: effects of task difficulty, type of processing, and practice. Cereb Cortex 7:374-385.

Goldman-Rakic PS (1995) Cellular basis of working memory. Neuron 14:477-485.

Gundel A, Wilson GF (1992) Topographical changes in the ongoing EEG related to the difficulty of mental tasks. Brain Topogr 5:17-25.

Haj-Dahmane S, Andrade R (1997) Calcium-activated cation nonselective current contributes to the fast afterdepolarization in rat prefrontal cortex neurons. J Neurophysiol 78:1983-1989.

Hockley WE, Murdock Jr BB (1987) A decision model for accuracy and response latency in recognition memory. Psyc Rev 94:341-358.

Huerta PT, Lisman JE (1996) Bidirectional synaptic plasticity induced by a single burst during cholinergic theta oscillation in CA1 in vitro. Neuron 15:1053-1063.

Iramina K, Ueno S, Matsuoka S (1996) MEG and EEG topography of frontal midline theta rhythm and source localization. Brain Topography 8:329-331.

Jensen O, Lisman JE (1996a) Novel lists of $7 \pm 2$ known items can be reliably stored in an oscillatory short-term memory network: interaction with long-term memory. Learn Mem 3:257-263.

Jensen O, Lisman JE (1996b) Hippocampal CA3 region predicts memory sequences: accounting for the phase precession of place cells. Learn Mem 3:279-287.

Jensen O, Idiart MAP, Lisman JE (1996) Physiologically realistic formation of autoassociative memory in networks with theta/gamma oscillations: role of fast NMDA channels. Learn Mem 3:243-256.

Joliot M, Ribary U, Llinas R (1994) Human oscillatory brain activity near $\mathrm{Hz}$ coexists with cognitive temporal binding. Proc Natl Acad Sci USA 91:11748-11751.

Jonides J, Schumacher EH, Smith EE, Koeppe RA, Awh E. ReuterLorenz PA, Marshuetz C, Willis CR (1998) The role of parietal cortex in verbal working memory. J Neurosci 18:5026-5034.

Klimesch W, Doppelmayr M, Schimke H, Ripper B (1997) Theta synchronization and alpha desynchronization in a memory task. Psychophysiology 34:169-176.

Krause CM, Lang AH, Laine M, Kuusisto M, Pörn B (1996) Eventrelated EEG desynchronization and synchronization during an auditory memory task. Electroencephalogr Clin Neurophysiol 98:319-326.
Lisman JE, Idiart MAP (1995) Storage of $7 \pm 2$ short-term memories in oscillatory subcycles. Science 267:1512-1514.

McElree B, Dosher BA (1989) Serial position and set size in short-term memory: the time course of recognition. J Exp Psychol 118:346-373.

McKone E (1995) Short-term implicit memory for words and nonwords. J Exp Psychol Learn Mem Cogn 21:1108-1126.

Mecklinger A, Kramer AF, Strayer DL (1992) Event related potentials and EEG components in a semantic memory search task. Psychophysiology 29:104-119.

Miller GA (1956) The magical number seven plus or minus two. Psychol Rev 63:81-97.

Monsell S (1978) Recency, immediate recognition memory, and reaction time. Cognit Psychol 10:465-501.

Nakamura K, Mikami A, Kubota K (1992) Oscillatory neural activity related to visual short-term memory in monkey temporal pole. NeuroReport 3:117-120.

Nicolelis MA, Baccala LA, Lin RC, Chapin JK (1995) Sensorimotor encoding by synchronous neural ensemble activity at multiple levels of the somatosensory system. Science 268:1353-1358.

O'Keefe J, Reece ML (1993) Phase relationship between hippocampal place units and the EEG theta rhythm. Hippocampus 3:317-330.

Pantev C, Makeig S, Hoke M, Galambos R, Hampson S, Gallen C (1991) Human auditory evoked gamma-band magnetic fields. Proc Natl Acad Sci USA 88:8996-9000.

Pavlides C, Greenstein YJ, Grudman M, Winson J (1988) Long-term potentiation in the dentate gyrus is induced preferentially on the positive phase of theta-rhythm. Brain Res 439:383-387.

Posner IP, Keele SW (1967) Decay of visual information from a single letter. Science 158:137-139.

Rahn E, Basar E (1993a) Prestimulus EEG-activity strongly influences the auditory evoked vertex response: a new method for selective averaging. Int J Neurosci 69:207-220.

Rahn E, Basar E (1993b) Enhancement of visual evoked potentials by stimulation during low prestimulus EEG stages. Int $\mathrm{J}$ Neurosci 72:123-136.

Ratcliff R (1978) A theory of memory retrieval. Psychol Rev 85:59-108.

Sarnthein J, Petsche H, Rappelsberger P, Shaw GL, von Stein A (1998) Synchronization between prefrontal and posterior association cortex during human working memory. Proc Natl Acad Sci USA 95:7092-7096.

Sasaki K, Tsujimoto T, Nishikawa S, Nishitani N, Ishihara T (1996) Frontal mental theta wave recorded simultaneously with magnetoencephalography and electroencephalography. Neurosci Res 26:79-81.

Shallice T, Vallar G (1990) The impairment of auditory-verbal shortterm storage. In: Neuropsychological impairments of short-term memory (Vallar G, Shallice T, eds), pp. 11-53. Cambridge, UK: Cambridge UP.

Singer W, Gray CM (1995) Visual feature integration and the temporal correlation hypothesis. Annu Rev Neurosci 18:555-586.

Smith EE, Chase WG, Smith PG (1973) Stimulus and response repetition effects in retrieval from short-term memory. J Exp Psychol 98:413-422.

Sternberg S (1964) Estimating the distribution of additive reaction-time components. Paper presented at Psychometric Society Meeting, Ontario, Canada, October.

Sternberg S (1966) High speed scanning in human memory. Science 153:652-654.

Tallon-Baudry C, Bertrand O, Delpuech C, Pernier J (1997) Oscillatory $\gamma$-band activity induced by a visual search task in humans. J Neurosci 17:722-734

Tallon-Baudry C, Bertrand O, Peronnet F, Pernier J (1998) Induced $\gamma$-band activity during the delay of a visual short-term memory task in humans. J Neurosci 18:4244-4254.

Tesche CD (1997) Non-invasive detection of ongoing neuronal population activity in normal human hippocampus. Brain Res 749:53-60.

Wehr M, Laurent G (1996) Odor encoding by temporal sequences of firing in oscillating neural assemblies. Nature 384:162-166. 